\title{
Using Frustration in the Design of Adaptive Videogames
}

\author{
Kiel M Gilleade, Alan Dix \\ Computing Department \\ Lancaster University \\ Lancaster, LA1 4YR, UK \\ +4401524 \{593419, 592268\} \\ gilleade@comp.lancs.ac.uk, \\ alan@hcibook.com
}

\begin{abstract}
In efforts to attract a wider audience, videogames are beginning to incorporate adaptive gameplay mechanics. Unlike the more traditional videogame, adaptive games can cater the gaming experience to the individual user and not just a particular group of users as with the former. Affective videogames, games that respond to the user's emotional state, may hold the key to creating such gameplay mechanics. In this paper we discus how the emotion frustration may be used in the design of adaptive videogames and the ongoing research into its detection and measurement.
\end{abstract}

\section{Keywords}

Adaptable, adaptive, affective computing, emotion, frustration, videogames.

\section{INTRODUCTION}

For videogames to achieve some degree of success in today's market they must appeal to as many players as feasibly possible. So designing games in a manner that provides the same user experience (i.e. entertaining) to all members of its audience irrespective of player motivation, experience or skill is becoming the focus of modern day games research. Traditional game design is well suited to covering particular clusters of players (e.g. hardcore, casual) as a developer's perception of what makes a good game is sure to appeal to someone. However, players who do not fall within these preconceived perceptions of the designer are likely to be alienated; they may initially be interested in the game's concept but are turned off by its execution (e.g. too easy, too hard).

\subsection{Adaptable Gameplay: Predetermined}

In the past designers have relied on the provision of adaptable gaming experiences to make for better audience coverage, for example most games come equipped with an easy, medium and hard difficulty setting. But while such experiences may offer better coverage it neglects the fact that the player is a dynamic entity and as such will absorb and adapt to that experience. The

Permission to make digital or hard copies of all or part of this work for personal or classroom use is granted without fee provided that copies are not made or distributed for profit or commercial advantage and that copies bear this notice and the full citation on the first page. To copy otherwise, or republish, to post on servers or to redistribute to lists, requires prior specific permission and/or a fee.

ACE 2004, June 3-5, 2004, Singapore.

Copyright 2004 ACM 1-58113-882-2/04/0006...\$5.00. designer will try to envisage such changes in the player and adapt the gameplay to reflect them so to ensure the game remains appealing throughout its usage. However, the variety of possible user responses means that some players will inevitably lie outside the scope of predetermined adaptation.

Imagine an action game. An inexperienced player, unfamiliar to this game and similar, takes half an hour to complete the first level. During play the user forms their own schemas relating to how the game and games like it function and so may tackle the next level in a slightly different fashion. The problem designer's face, is in determining how much the player advances their schemas during that first level because in order for the next and successive levels to be appealing they need to be adapted to reflect that advancement in their design.

Many games view player advancement as linear and so become progressively more complex (e.g. difficult) as the game is traversed. Unfortunately this view is that of an 'ideal' user and so some players may feel somewhat discontented when they advance in a manner that is counter to the 'ideal' advancement model and so the game loses it appeal. But alternatively designing a videogame to cater for all levels of advancement using traditional theory would become bloated and most likely not appeal to anyone.

This form of predetermined adaptability is also found in nongame interactive systems. Many applications have profiles or preferences, some of which are to do with technical parameters (e.g. machine names, directories etc.), but some to do with user's preferences (e.g. font colours, 'intelligent' formatting, etc.). Some applications have several 'levels', not unlike game difficulty levels. More expert users will see more menu options but novice users have some options hidden, so have less to choose from and are unlikely to make silly mistakes! This builds on the Carroll and Carrithers training wheels [3] concept except that in this early work the novice menu was complete but with 'expert' options grayed out. In the user interface the aim is to help the user to avoid making mistakes and know what is possible. In games of course suspense and excitement require different adaptations.

\subsection{Adaptive Gameplay: Dynamic}

Preferably videogames should be capable of dynamically changing their design (i.e. adaptive) in light of the player's ongoing interactions with the videogame. Therefore making the 
gaming experience fit the individual user ${ }^{1}$. However creating adaptive gameplay ${ }^{2}$ is not something to be taken lightly. We need to consider the motivation of the users: why they want to play, their experience and skills: how able are they to play, and detection: how to identify when change is necessary.

\subsubsection{Physiological Motivators}

Why does the user want to play? Is it for the challenge, the fun of it all or perhaps it's a displacement activity? Going back to our imaginary game, suppose the end-of-game boss has defeated the player multiple times in succession. Repetitive actions such as these become frustrating after a while as players would ideally like to see a resolution being so close to completing the game. For an adaptive game the simplest solution would be to incapacitate the boss in some fashion that makes it easier to defeat. But should the user be playing for the challenge it would be akin to saying "your not good enough, I'll let you win on purpose". A challenge orientated player derives satisfaction from completing particular difficult challenges, if we reduce the challenge when they fail, that satisfaction is removed and they will not thank you for doing so.

In order for adaptive games to make the kind of changes that are 'motivational correct' for the player we need to understand the primary psychological motivators that govern that player.

\subsubsection{Experience / Skill}

How strong is the user? A videogames audience is invariably filled with a multitude of different strength players. Suppose the player still continues to fail in defeating the end-of-game boss in our imaginary game. Motivational issues aside, the game could be designed to respond by lowering the boss's difficulty. However this raises the question; what level of change should be applied?

Clearly the skill and experience of users will be an important factor in deciding that level (e.g. a strong player would only require a minor drop in the boss's difficulty in order to achieve success). However the focus of this paper will be on detecting that change is required, so we will not discuss further the precise nature of these changes.

\subsubsection{Detection}

In order to realise adaptive gameplay we need suitable methods of detecting when change is needed. To some extent the movement from one level to the next is a form of detection - the player's past performance is used to suggest a reasonable time to change the games design. However, this does not easily distinguish 'skin of the teeth' successes from easy ones, the former of which may be better served by continuing play at a similar setting and the latter by advancing the games difficulty. Consequently we would like to detect the feelings of the player and use this to determine appropriate points of change as it can provide more meaningful data.

\footnotetext{
${ }^{1}$ Splinter Cell: Pandora's Tomorrow (Ubisoft, 2004) is reputed to include an ambient AI [14] that provides some measure of adaptive play.

${ }^{2}$ This is not the same as allowing for adaptive gameplay as with multiplayer games. Although human opponents have the potential to adapt it doesn't mean they will.
}

Subjective feelings are precisely the focus of affective computing research: "computing that relates to, arises from, or deliberately influences emotions" [10]. By reacting to the user's own emotions, games can be made to adapt to suit their particular style of play. In this paper we discuss how the emotion frustration may be used as an indicator for change in the design of adaptive games and the ongoing research into its detection and measurement.

\section{VIDEOGAME FRUSTRATION}

Playing videogames can sometimes be somewhat frustrating. For example repeatedly failing to defeat a particular enemy or wandering aimlessly around a maze-like dungeon looking for the exit. Such situations as these are all liable to frustrate the player if they aren't revolved in a reasonable period of time, but why is that? What is frustration?

Frustration is that which arises when the progress a user is making towards achieving a given goal is impeded. It is a negative emotion and if monitored for can be used to indicate when a user is in need of assistance. Therefore the manner of games we could create using frustration as an indicator for change, would be those that assisted the player in tackling situations they deem too difficult to handle by themselves, as they arise. This offers games the chance to avoid those situations that under normal conditions would give cause for the user to discontinue play.

\subsection{D Frustration}

For a human to recognise a frustrated player is not difficult; the tension in the face, the verbal abuse thrown at the screen, the violence carried out on the gamepad. These are common physical manifestations of frustration a human may perceive when play doesn't exactly go to plan. A particular apt human might even be able to identify the source of the player's frustrations. However an automated system may not be capable of correlating those signs a human uses to recognise frustration with its source with the same ease a human does. This is critical to the design of an adaptive game using frustration as an indicator for change, without knowing the source of the player's frustrations a suitable response (i.e. change to game design) cannot be formulated.

Therefore in order to use frustration successfully as an indicator for change, a better understanding of its dimensions from a gaming context is required. We have identified two such dimensions, which we call at-game and in-game frustration.

\subsubsection{Physical Failures}

Videogames often require players to input time-sensitive command sequences in order to gain a tactical advantage over their opponent(s). From the trademark fireball maneuver of fighting games (see Fig 1) to the single button special bomb attack found in shooting games. Actions such as these give players the potential to progress against a computer or human opponent. Being unable to complete a command gives cause for the player to become frustrated as they would not be able to compete in a manner that was fair. 


\section{$\downarrow \forall \Rightarrow+P$}

Figure 1: Down, Down Right, Right and Punch; execute this maneuver when facing towards your opponent in a one-on-one fighting game and a fireball is usually not too far behind.

For example in Killer Instinct (Rare, 1994), a one-on-one fighting game, players can perform powerful combo attacks if they input the correct command sequence. Combo attacks can drain an opponent's energy much more than a single button attack. But combo attacks can be countered with another command sequence should they have connected. If a strong and weak player face each other, although the weak player maybe be able to perform a limited number of combo attacks the strong player is certainly going to break them with a counter. However a weak player is unlikely to break from a strong player's combo attack, which is ultimately going to be more devastating. The weak player is not going to be able to compete should this continue and so gives cause for them to become frustrated.

Given these kind of conditions we call this at-game frustration.

At-game frustration is that which arises from a failure to operate the input device (e.g. gamepad, keyboard, joystick) in a manner that would give the player the potential to progress (i.e. compete). Contributing factors include; how commands are laid out over the input device, responsiveness of the input device, the dimensions of the command sequence (e.g. number of commands involved, allotted time to input command sequence) and the skill of the player.

This at-game frustration is similar to the concept of breakdown in user-interface design. The concept of breakdown stems from Heidegger $^{3}$ and relates to that moment when a tool in some way ceases to be invisible, instead of invisibly being used to accomplish a purpose it becomes the focus of attention. In a computer system when the user has to focus on the interface rather than the task at hand this is breakdown. In 'work' interfaces this breakdown is always viewed as bad, however, in playful or ludic designs breakdown may be deliberate in order to encourage reflection or experimentation [7]

Similarly, in virtual reality there is normally an aim to achieve immersion, to lose yourself in the virtual experience and become unaware of the VR hardware around you. However, here there are exceptions too. For example, in the use of virtual reality to treat phobias, patients are exposed to virtual experiences that are intended to be like those that frighten them: spiders, heights, crowds. These work because they are realistic, but the patient is also aware that it is 'just a simulation' and is able to cope [9].

In games, the intention is usually to get through the controller and the hardware and be in the game, what in virtual reality research is called engagement. So at-game frustration will almost always correspond to a failure in the game. However there will be exceptions, for example, the taking over of the controller by

\footnotetext{
${ }^{3}$ Selected aspects of Heidegger's phenomenological philosophy have been influential in several areas of Human-Computer Interaction, and is discussed, for example, in Dourish's "Where The Action Is" [4].
}

Psycho Mantis in Metal Gear Solid (Konami, 1998) creates a 'breakdown', but by doing this makes the real world become part of the game, perversely making the whole experience more immersive.

\subsubsection{Mental Failures}

Videogames require players to complete one or more objectives in order to progress to the next challenge. For example in Doom (id Software, 1993), in order to progress to the next level (challenge) the player has to reach the exit. To reach the exit the player must; explore the game world, find door keys, avoid or eliminate monsters and above all else survive with some health intact (objectives). When an objective is lost the player can not proceed to the next challenge and so gives cause for the player to become frustrated.

Lost in this context can either mean literally (player fails to recognise or remember the clues denoting the challenge objective(s)), when an objective is not given and the user is meant to explore the game world in search of the challenge's objective(s) or when an objective is given but the means to resolve it is lost. For example in Metal Gear Solid (Konami, 1998), a sniper rifle is required to defeat Sniper Wolf. The player is told to search for this weapon, but isn't told its location upfront. There is no means to resolve this objective unless the player wishes to search the entire game world for the rifle. Exploration such as this can be frustrating if not resolved within a reasonable time frame.

Given these kind of conditions we call this in-game frustration.

In-game frustration is that which arises from a failure to know how a challenge is to be completed. Contributing factors include; size of the game world, complexity of the game dialogue and world design (e.g. if visual clues are used to infer the existence of something hidden are they sufficient enough to distinguish from the surroundings).

Using these two dimensions we can design experiments to investigate the correlation between the various physical manifestations of frustration and its respective source.

\section{DETECTING FRUSTRATION}

Physiological measures such as blood pressure, heart rate and skin conductivity are amongst some of the most commonly used indicators of arousal. In affective computing, emotions are commonly inferred through arousal. So, should we wish to use frustration in adaptive games, it would make sense to follow on from the research already conducted.

However as argued by Sykes et al in Affective Gaming: Measuring emotion through the gamepad [13], physiological measures of arousal are open to corruption when used in the traditional gaming environment. For example if a user is playing Doom (id Software, 1993), what we call a reflex game (as it requires fast muscle reflexes to play), the stress of interacting is in all likelihood going to influence the physiological measures normally used to infer emotion (e.g. skin conductance). This would paint a disproportionate view of the player's affective state.

Consequently if we want to detect frustration in our games we are going to have to use alternative methods. As a human doesn't necessarily use such intimate measures to judge someone else's emotions this shouldn't be too much of a problem. For example we could use facial expressions as these have been long associated 
with denoting certain emotional states [5]. But this would require adding additional artifacts such as a camera to the player's physical gaming environment (e.g. bedroom).

For games that require the use of an additional artifact (e.g. EyeToy games) this is not a concern, but to ask players to at all times remain in contact with that artifact (e.g. stay within the cameras field of view during play) in a traditional gaming environment so it can gauge their frustration is ludicrous. No one or at least very few people would accept the technology. A reasonable solution would be to detect frustration through the existing technology framework, in this case the input device (e.g. gamepad, keyboard, joystick) and the game itself (e.g. progress made in the game).

\subsection{Experiment Setup}

There is currently very little research regarding 'affect' and how it may be inferred from the various attributes of the input device. Although research outside the gaming context is available it may be unsuitable for specific use in videogames because the interpretation of measures (e.g. arousal) used to infer emotion is influenced by the situational context [11]. The experiment conducted by Sykes [13] indicates it is possible to measure the player's level of arousal by monitoring button pressure on the gamepad but there isn't much work that goes beyond (e.g. infers an emotion). At Lancaster University we are in the process of devising a series of experiments that would provoke at-game and in-game frustration during play on a videogame console (input device is therefore a gamepad), so the resultant user behavior can be recorded.

Based on informal observations we have already made on frustrated players our experiments will be monitoring; error rates (is an illogical button sequence entered? ${ }^{4}$ ), button pressure, gamepad grip, gamepad tilt, vibration (is the gamepad being shaken?), swing (is the gamepad being moved in a direction reminiscent of the player avatar) and game progression (is an objective taking longer than expected to complete?). It is expected that the differences in the causes of frustration shall generate distinctive patterns in the collected data. For example we suspect disjointed avatar movement would indicate at-game frustration whereas a lack of game progression coupled with an increase in gamepad grip might indicate in-game frustration.

The experiment is expected to be completed within the coming year and should provide the necessary data to create adaptive games of the sort that assist problematic play using the player's frustration as a point of reference for change.

\section{DISCUSSION}

In creating emotional experiences in traditional videogames (i.e. non-adaptive), frustration is sometimes deemed necessary to heighten the overall experience [6]. But with frustration being a negative, designers have to take care not to push their luck as deliberately inciting frustration for the wrong period of time can have the opposite effect (e.g. too short and the experience the

\footnotetext{
${ }^{4}$ Silent Hill (Konami, 1999) a survival horror game, may cause the player to jump and reflexively press an odd combination of buttons when a 'scary' event occurs. Although these commands don't add to play they could be used to infer emotion.
}

designer was aiming for can't be realised, too long and the player may give up and turn to other pursuits). Being able to detect frustration, if possible, is therefore not just for assisting problematic play. It can be used to weave much more complex emotional experiences into game stories; as in the previous example detection could determine what period of time is right for the individual user. Game evaluation could also benefit, for example if the players frustration level can be measured it may be possible to identify problematic game designs before the game is released.

The role affective technologies will play in the gaming industry is certain to create some new and exciting user experiences, such as the therapeutic (i.e. biofeedback), where games are designed to promote a healthier mental well being [2, 12]. For example SMART (Self Mastery And Regulation Training) [12], a tool designed to treat attention deficit hyperactivity disorder in children inhibits the gamepad when playing off-the-shelf videogames such as Tony Hawk's Pro Skater whenever the player becomes distracted. Creating more emotionally realistic experiences is also a possibility. For example in AffQuake [1], an affective version of Quake 2 (id Software, 1997), the player's avatar can be made to jump back in response to the player being startled by events in the game (e.g. sudden appearance of an enemy).

However affective research has a tendency to deal with 'ideal' situations, where the environment experiments are conducted in is devoid of the usual factors that would distort the results. Tokimeki Memorial Oshiete Your Heart (Konami, 1997), a Japanese arcade dating simulator that uses a players pulse and sweat level to influence the outcome of a date is about as easy to corrupt as taking a jog before play (excess sweat distorts physiological measures). Research experiments rarely have to consider the implication of outside factors as its easier to prove a point without them, however if we want to create adaptive games based on the player's affective state, in this instance frustration, this is not an option as distortions to the affective state without compensation will detract from the games appeal.

In a previous project of ours, the Intelligent Gaming System (IGS) [8]; a development platform for creating affective videogames, we designed several methods of measuring the player's level of enjoyment based on gaming experience. Games built using IGS used this measure to instrument changes to the gameplay in order to keep play stimulating for that particular user. During initial trails we found that when the agent responsible for selecting game changes was given a modus operandi that conflicted with the player's experience, the point of reference by which changes were made became invalid and so proved detrimental to a games appeal.

It is therefore important that the next tentative steps towards adaptive games based on the player's affective state are taken with care.

\section{REFERENCES}

[1] AffQuake. Retrieved February 2004 from http://affect.media.mit.edu/AC_listings.html

[2] Bersak, D., McDarby, G., Augenblick, N., McDarby, P., McDonnell, D., McDonal, B., Karkun, R. Biofeedback using an Immersive Competitive Environment. Online Proceedings 
for the Designing Ubiquitous Computing Games Workshop, Ubicomp 2001.

[3] Carroll, J. M., Carrithers, C. Blocking Learner Errors in a Training Wheels System. Human Factors, 26:377-389, 1984.

[4] Dourish, P. Where The Action Is: The Foundations of Embodied Interaction. MIT Press (2001).

[5] Ekman, P. Facial Expression and Emotion. American Psychologist (1993), 48(4) p384-392.

[6] Freeman, D. Creating Emotions in Games. New Riders (2003), p185.

[7] Gaver, B., Beaver, J., Benford, S. Ambiguity as a Resource for Design. In Proceedings of CHI 2003. New York, USA: ACM Press.

[8] Gilleade, K., Allanson, J. A Toolkit for Exploring Affective Interface Adaptation in Videogames. Volume 2 of Proceedings of HCI International 2003, pp. 370-374.

[9] Hodges, L.F., Anderson, P., Burdea, G., Hoffman, H.G. and Rothbaum, B.O. VR as a Tool in the Treatment of
Psychological and Physical Disorders. IEEE Computer Graphics and Applications (2001), 21(6), p25-33

[10] Picard, R. Affective Computing. MIT Press (1997).

[11] Schachter, S. The Interaction of Cognitive and Physiological Determinants of Emotional State. Advances in Experimental Psychology Volume 1. Academic Press New York (1964), p49-80.

[12] S.M.A.R.T. Brain Games. Retrieved February 2004 from http://www.cyberlearningtechnology.com

[13] Sykes, J., Brown, S. Affective Gaming: Measuring Emotion Through the Gamepad. Conference on Human Factors in Computing, CHI 2003, p732-733.

[14] Ward, M. Computer Games Learn New Tricks. BBC News Online, December 2003. Retrieved February 2004 from http://news.bbc.co.uk/1/hi/technology/3257458.stm 